\title{
EARLY RESULTS FROM THE INFRARED SPECTROGRAPH ON THE SPITZER SPACE TELESCOPE
}

\author{
J. R. Houck ${ }^{1}$, V. Charmandaris ${ }^{1}$, and B. R. Brandl ${ }^{2}$ \\ ${ }^{1}$ Cornell University, Space Sciences, Cornell University, Ithaca, NY 14853, USA \\ ${ }^{2}$ Leiden Observatory, Niels Bohrweg 2,\#535, P.O. Box 9513, 2300 RA Leiden, Nether- \\ lands \\ jrh13@cornell.edu,vassilis@astro.cornell.edu,brandl@strw.leidenuniv.nl
}

\begin{abstract}
The Infrared spectrograph, IRS, on the Spitzer Space Telescope has greatly improved the sensitivity in the thermal infrared and opened many new avenues in infrared astronomy over the range of 5.5 to 38 microns. With the IRS an object with midinfrared flux of $\sim 1$ mJy can produce a usable low resolution spectrum in an hour of telescope time. The midinfrared study of star formation at low metallicity has been enabled by this new sensitivity.
\end{abstract}

\section{Introduction}

The infrared spectrograph (IRS; Houck et al. 2004a), one of the instruments in the Spitzer Space Telescope (Werner et al. 2004) the fourth and last of NASA's Great Observatories, was launched into orbit about the Sun on August 25, 2003. Routine observations began in December 2003, with all of the instruments performing extremely well. The IRS consists of four individual spectrographs that are identified by their wavelength range and spectral resolution: Short-Low (SL, 5.5-14.5 $\mu \mathrm{m} ; R=$ $\lambda / \delta \lambda \sim 90$ ), Short-High(SH, 9.9-19.6; $R=600$ ), Long-Low (LL,14.0-38; $R=90$ ), and Long-High (LH, 18.7-37; $R=600$ ). The widths of the entrance slits are set to $\lambda_{\max } / D$ where $\lambda_{\max }$ is the longest usable wavelength for each module and $D=85 \mathrm{~cm}$, the telescope aperture. The slits lengths vary from 11.8 to 151.3 arcsec. The slit widths are imaged onto two detector pixels. The low resolution slits are as long as possible given the limited space available. The high resolution modules are crossed echelle spectrographs with slit lengths of 6 pixels. In addition the IRS SL module has two imaging apertures $(\sim 60 \times 90$ arcsec $)$ with bandpass 
centers at $16 \mu \mathrm{m}$ and $22 \mu \mathrm{m}$. These are used for direct imaging and target acquisition. The detectors are $128 \times 128$ arrays of Si:As (SL and $\mathrm{SH})$ and $\mathrm{Si}: \mathrm{Sb}(\mathrm{LL}$ and $\mathrm{LH})$ with pixel sizes of $75 \times 75 \mu \mathrm{m}$. There are no moving parts in the IRS. Additional information on the design and operation of the IRS can be found in Chapter 7 of the Spitzer Observers Manual, http://ssc.spitzer.caltech.edu/documents/SOM/, and at the IRS Science Center, http://isc.astro.cornell.edu/.

Nearly 100 science papers based on the early observations from Spitzer have been published in a special issue of the Astrophysical Journal Supplements (vol.154, 1 Sep 2004). In this paper we discuss the early results on the study of star formation in a very low metallicity blue compact galaxy, SBS 0335-072 (Houck et al. 2004b).

\section{Observations}

We chose SBS 0335-052, along with approximately 20 other well studied BCDs, to investigate the characteristics of star formation at very low metallicities using the IRS (Houck et al. 2004a) on Spitzer (Werner et al. 2004). Similar low metallicity objects may be detected at much higher luminosity and much greater redshifts in Spitzer discovery surveys.

SBS 0335-052 was observed using both IRS low resolution modules. The spectrum extends from 5.3 to $35 \mu \mathrm{m}$ and was obtained on 6 Feb 2004 . The red peak-up camera in medium-accuracy mode was used to locate the mid-IR centroid of the source and move it to the center of the spectrograph slits. The total integration time was 42 minutes with 28 minutes for the Short-Low module and 14 minutes for the Long-Low module. The total elapsed time including the telescope slew, target acquisition, settling, array conditioning, and integration was 61.3 minutes.

The basic processing of the data, such as ramp fitting, dark sky subtraction, removal of cosmic rays, droop and linearity correction, wavelength calibration, etc, was performed using the IRS pipeline at the Spitzer Science Center (version S9.1). The resulting spectral images were sky-subtracted and a one-dimensional spectrum then extracted. The peak-up images were also used to derive a photometric point at $22 \mu \mathrm{m}$ (filter bandwidth 18.5-26.0 $\mu \mathrm{m}$ ). As described in detail in chapter 7 of the Spitzer Observer's Manual, during IRS peak up we obtain 6 images of the science target. The on source time for the $22 \mu \mathrm{m}$ peak-up images of our target was $6 \times 8=48$ s and each image was created by reading the array in double-correlated sample mode. We processed the data on the ground to remove cosmic rays and the residual noise of the electronics. The resulting image had a prominent diffraction ring and was indistinguishable from the image of a point source. The conversion 


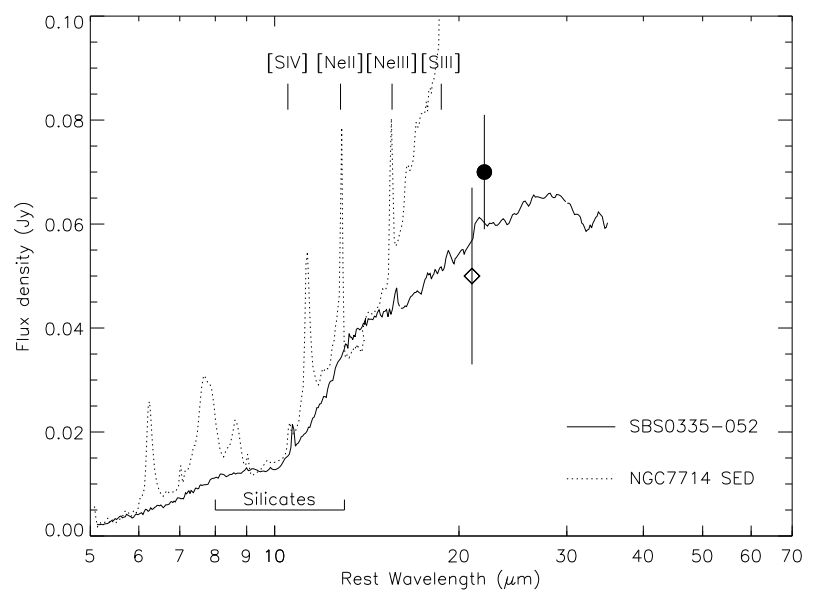

Figure 1. The IRS low resolution spectrum of SBS 0335-052 (solid line) along with the spectrum of NGC 7714 (dotted line) from Brandl et al. The spectrum of NGC 7714 has been divided by 9.53 so that its $14 \mu \mathrm{m}$ flux density matches that of SBS 0335052. Note the complete lack of PAHs in the spectrum of SBS 0335-052 as well as the spectrum peak at $\sim 30 \mu \mathrm{m}$. The solid circle is our $22 \mu \mathrm{m}$ peak-up photometric point, while the diamond corresponds to the $21 \mu \mathrm{m}$ Gemini point from Plante \& Sauvage.

to flux density was based on a number of calibration stars for which peak-up images, IRS spectra, and reliable templates are available (Cohen et al. 2003). We find that the $22 \mu \mathrm{m}$ flux density of SBS 0335-052 is $70 \pm 11 \mathrm{mJy}$.

\section{Interpretation}

Figure 1 shows the 5.3-35 $\mu \mathrm{m}$ spectrum of SBS 0335-052 as observed by the IRS. Our data are in good agreement with the overall shape and intensity of the $5-15 \mu \mathrm{m}$ ISO spectrum of Thuan et al. (1999), but our signal to noise is at least a factor of 10 higher. This enables us for the first time to directly detect a few mid-IR ionic lines, while placing strong upper limits on others. The $9.7 \mu \mathrm{m}$ silicate absorption feature is clearly evident in our spectrum, and the $18 \mu \mathrm{m}$ feature is probably present.

In Figure 1 we also present a scaled version of the IRS low-resolution spectrum for the prototype starburst nucleus of NGC 7714 (Brandl et al. 2004), normalizing its flux to the corresponding flux of SBS 0335052 at $14 \mu \mathrm{m}$ (the actual flux for NGC 7714 is $\sim 9.5$ times larger than shown). As also noted by Thuan et al. (1999), a striking difference between the spectrum of SBS 0335-052 and that of a more typical starburst is the absence of polycyclic aromatic hydrocarbon (PAH) features and 
low excitation ionic lines. In starburst galaxies, emission from PAHs is thought to originate from the photodissociation envelopes bordering the H II regions produced by the ionizing starburst. How these features can be absent in a low-metallicity starburst is an important astrophysical question. One possibility is that the absence of PAHs is due to low abundance of carbon and/or nucleating grains; another possibility is that PAHs are quickly destroyed.

Table 1. Mid-IR Line Fluxes of SBS 0335-052

\begin{tabular}{|c|c|c|}
\hline Ion & $\lambda(\mu \mathrm{m})$ & $\overline{\text { Flux }\left(\times 10^{-17} \mathrm{Wm}^{-2}\right)}$ \\
\hline$[\mathrm{S}$ IV] & 10.51 & $1.62 \pm 0.09$ \\
\hline$[\mathrm{S}$ III $]$ & 18.71 & $<0.54$ \\
\hline$[\mathrm{Ne} I \mathrm{II}]$ & 12.81 & $<0.28$ \\
\hline$[\mathrm{Ne}$ III $]$ & 15.55 & $1.40 \pm 0.08$ \\
\hline $\mathrm{H}_{2} 0-0 \mathrm{~S}(3)$ & 9.67 & $<0.37$ \\
\hline
\end{tabular}

Table 1 shows that the observed line ratios of $\log ([\mathrm{S} \mathrm{IV}] /[\mathrm{S} \mathrm{III}])>0.48$, and $\log ([\mathrm{NeIII}] /[\mathrm{Ne} \mathrm{II}])>0.69$ are similar to the most extreme example of ultra-compact H II regions in our Galaxy. The ratios indicate that the radiation field is extremely hard and corresponds to an effective stellar temperature of $T_{\text {eff }} \geq 4 \times 10^{4} \mathrm{~K}$, assuming solar abundance (see Martin-Hernandez et al. 2002). This would suggest that the absence of PAHs results from their destruction by the hard UV photons and strong winds produced by the massive stars (i.e. Allain et al. 1996). Such a scenario is quite likely since in low metallicity systems the attenuation of UV photons is small and consequently their mean free path in the interstellar medium can be larger and photodissociation of PAHs may occur over considerably larger scales than those seen in typical Galactic $\mathrm{H}$ II regions. Whatever, the PAH life-cycle is in SBS 0335-052, the primary result is the well-defined absence of these features in the midIR. (Our recent results indicate a strong correlation between the PAH strength and the metallicity of the host BCD.)

Another major difference between the two spectra shown in Figure 1 is that in SBS 0335-052 the continuum shortward of $\sim 15 \mu \mathrm{m}$ appears similar to that which underlies the emission features in NGC 7714. However, these continua depart dramatically at longer wavelengths. The continuum of NGC 7714 increases rapidly at longer wavelengths because of a massive cool dust component which characterizes many luminous infrared galaxies. Conversely, the spectrum of SBS 0335-052 peaks at $28 \mu \mathrm{m}$. In Figure 2 we have drawn an offset power-law, $f_{\nu} \sim \nu^{1.3}$, to the IRS spectrum to extrapolate to longer wavelengths. Using the non-thermal $1.46 \mathrm{GHz}$ flux density of the galaxy measured by Hunt et 


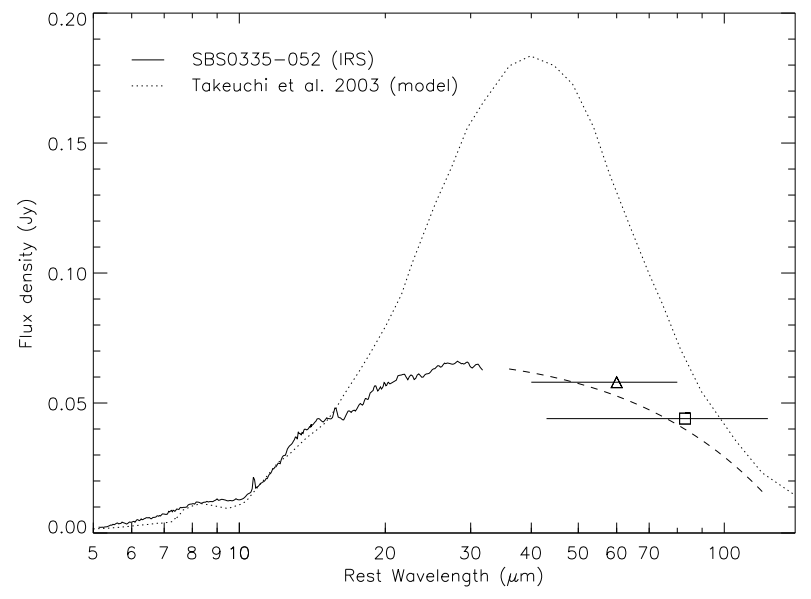

Figure 2. The IRS spectrum of SBS0335-052 (solid line) along with the model of Takeuchi et al. (dotted line). Based on the observed 5-35 $\mu \mathrm{m}$ SED of the galaxy we can extrapolate to longer wavelengths (dashed line). The difference between the observed SED and the model has only a small effect in the infrared luminosity, but it substantially reduces the amount of cold dust in the galaxy. We also show estimates derived from the radio data of the average flux density over the far-infrared range and the upper limit at $60 \mu \mathrm{m}$ (box and triangle, respectively).

al. (2004) and the radio to far-infrared correlation, we predict that the far-infrared luminosity is $F_{\mathrm{FIR}}(43-123 \mu \mathrm{m})=2 \times 10^{-15} \mathrm{Wm}^{-2}$. The corresponding average flux density over this wavelength range is $\sim 44 \mathrm{mJy}$. Even if the entire far-infrared luminosity originates from the $60 \mu \mathrm{m}$ band, the corresponding flux density would be $58 \mathrm{mJy}$, well below the Plante \& Sauvage (2002) $112 \mathrm{mJy}$ measurement at $65 \mu \mathrm{m}$. However, the $58 \mathrm{mJy}$ estimate is consistent with the IRS spectrum which clearly decreases at wavelengths longer than $30 \mu \mathrm{m}$.

These two major differences, no PAH features and relatively flat continuum, provide an initial indication that the PAH criterion for identifying starbursts may not apply to systems of low metallicity. Mid-IR spectra of Active Galactic Nuclei (AGN) often display ionic lines and also lack PAH emission features, but the continua are typically even flatter between 5-20 $\mu \mathrm{m}$ than SBS 0335-052 (see ISO observations of Seyfert 1s by Clavel et al. 2000, or the nuclear spectrum of NGC 1068 by Sturm et al. 2000 and Le Floc'h et al. 2001 as well as Peeters et al. 2004). If metal poor galaxies such as SBS 0335-052 can be present at $z \sim 0.06$, it is likely that similar systems may exist not only as high- $z$ primordial galaxies, but also at intermediate redshifts $0.5<z<1.5$ where moderate luminosity galaxies $\left(L_{\mathrm{IR}}<10^{11} L_{\odot}\right)$ are accessible with the deep 
infrared surveys performed by Spitzer. Far-infrared and submillimeter photometric methods for estimating redshifts based on spectral energy distributions (SEDs) of nearby metal rich systems, with continua peaking at $\sim 100 \mu \mathrm{m}$, would fail to identify metal poor systems with SEDs similar to that of SBS 0335-052, which peaks at $\sim 28 \mu \mathrm{m}$.

\section{Conclusions}

The very low metallicity Blue Compact Dwarf galaxy SBS 0335-052 is shown to have a very unusual spectrum which is quite different from the spectrum of typical starburst galaxies: the flux density, $f_{\nu}$, peaks at $\sim 28 \mu \mathrm{m}$ while the luminosity, $\nu f_{\nu}$, peaks at $\sim 20 \mu \mathrm{m}$. There are no detectable PAH emission features. The spectrum is characterized by a warm $(\sim 150 \mathrm{~K})$ dust component with a more massive cool $(\sim 65 \mathrm{~K})$ dusty envelope. However, the mass of the cool region is far less than what had been previously estimated. Silicate absorption features are clearly present $\left(A_{9.7 \mu \mathrm{m}} \geq 0.49 \mathrm{mag}\right)$. Preliminary analysis of the infrared ionic lines suggests that the young central cluster may have already polluted the cocoon enshrouding it, and its metallicity could be higher than that determined from optical observations alone. Upcoming midIR spectroscopy using the high resolution modules of IRS will provide higher sensitivity and more accurate line fluxes.

\section{References}

Allain, T., Leach, S., \& Sedlmayr, E. 1996, A\&A, 305, 602

Brandl, B. R., et al. 2004, ApJS, 154, 188

Clavel, J., et al. 2000, A\&A, 357, 839

Cohen, M., Megeath, T.G., Hammersley, P.L., Martin-Luis, F., \& Stauffer, J. 2003, AJ, 125, 2645

Houck, J. R., et al., 2004a, ApJS, 154, 18

Houck, J. R., et al., 2004b, ApJS, 154, 211

Hunt, L. K., Dyer K. K., Thuan, T. X., \& Ulvestad, J. S. 2004, ApJ, 606, 853

Le Floc'h, E., Mirabel, I. F., Laurent, O., Charmandaris, V., Gallais, P., Sauvage, M.,

Vigroux, L., \& Cesarsky, C. 2001, A\&A, 367, 487

Martin-Hernandez, N. L., et al. 2002, A\&A, 381, 606

Peeters, E., Spoon, H.W.W., \& Tielens, A.G.G.M., 2004, ApJ, accepted [astro-ph/0406183]

Plante, S. \& Sauvage, M. 2002, AJ, 124, 1995

Rees, M. J. 1998, Space Science Reviews, 84, 43

Sturm, E., Lutz, D., Tran, D., Feuchtgruber, H., Genzel, R., Kunze, D., Moorwood,

A. F. M., \& Thornley, M. D. 2000, A\&A, 358, 481

Thuan, T. X., Sauvage, M., \& Madden, S. 1999, ApJ, 516, 783

Werner, M., et al. 2004, ApJS, 154, 1 